\title{
The Minimally Invasive Retroperitoneal Transpsoas Approach
}

\author{
Tien V. Le and Juan S. Uribe \\ Department of Neurosurgery and Brain Repair, \\ University of South Florida, Tampa, Florida,
}

USA

\section{Introduction}

Minimally invasive spine surgery has evolved from traditional open spine surgery, and it is an accepted, safe alternative (McAfee, et al., 2010). Traditional open operations for lumbar interbody fusion include anterior lumbar interbody fusion (ALIF), posterior lumbar interbody fusion (PLIF), and transforaminal lumbar interbody fusion (TLIF). The ALIF provides for a large interbody graft for disc space re-expansion, restoration of lumbar lordosis, and elimination of discogenic pain (Hodgson \& Stock, 1956). In addition, posterior facet joint complexes and tension bands remain intact. However, an access surgeon may be needed, and complications can include a risk of vascular injury and also rare iatrogenic retrograde ejaculation in males postoperatively. The TLIF (Harms \& Rolinger, 1982; Harms \& Jeszenszky, 1998) was developed as a modification of the PLIF (Cloward, 1953) to decrease the degree of nerve root and thecal sac manipulation, and it allows for interbody fusion, concurrent posterior segmental instrumentation, and circumferential fusion. It can be performed either in an open or minimally invasive manner. The graft size is typically smaller than that of the ALIF, however.

First introduced by Luiz Pimenta in 2001, the retroperitoneal transpsoas minimally invasive lateral interbody fusion (MIS LIF) is a safe and effective alternative to anterior or posterior approaches for lumbar fusion (Pimenta, 2001; Ozgur, et al., 2006). Advantages include indirect neurological decompression with less tissue trauma, minimal blood loss, shorter operation times, less wound issues, placement of a larger cage, and early patient mobilization (Eck, et al., 2007; Benglis, et al., 2008; Wang, et al., 2008; Uribe, et al., 2010). In addition, normal stabilizing ligaments are not sacrificed as compared to other interbody techniques.

This technique was an adaptation of an endoscopic lateral transpsoas approach to lumbar fusion as described by Bergey et al. (Bergey, et al., 2004). They found that the endoscopic lateral transpsoas approach to the lumbar spine was a safe method to fuse the lumbar vertebrae, which allowed for exposure of the lumbar spine without mobilization of the great vessels or sympathetic plexus.

Today, there are several systems from various manufacturers that will allow for an MIS lateral retroperitoneal transpsoas approach. The two most common are the eXtreme Lateral Interbody Fusion/XLIF® (NuVasive, San Diego, CA) and Direct Lateral Interbody Fusion/DLIF® (Medtronic, Memphis, TN). 
Clinical applications of the retroperitoneal transpsoas MIS LIF include a wide range of spinal conditions including trauma, adult degenerative scoliosis, degenerative disc disease, spondylosis with instability, lumbar stenosis, spondylolisthesis, tumor, and adjacent segment failure. Research on MIS LIF is very active, and clinical outcomes appear to be promising.

\section{Anatomic considerations}

The lateral approach may be unfamiliar to spine surgeons who are accustomed to a posterior approach. Because of this, a review of key anatomic structures encountered with the lateral approach is paramount. In the order encountered, the muscles include the external oblique, internal oblique, and the transversus abdominis muscle. Once the retroperitoneal space is entered, the quadratus lumborum and psoas muscle are then encountered. The details of blunt dissection, as opposed to electrocautery, are discussed later, but careful attention must be paid in order to avoid injuring a traversing lumbar plexus nerve, which could lead to postoperative deficits.

\subsection{The lumbar plexus}

The lumbar plexus is found within the substance of the psoas muscle. It is a part of the lumbosacral plexus, and it is made of the primary ventral rami of the first four lumbar nerves and a contribution of the subcostal nerve (T12), the last thoracic nerve. Multiple motor and sensory nerves are given off. The major motor branches consist of the femoral (L2-4) and obturator (L2-4) nerves. The major cutaneous, sensory branches consist of the iliohypogastric (L1), ilioinguinal (L1), genitofemoral (L1-2), lateral femoral cutaneous (L2-3), and anterior femoral cutaneous (L2-4) nerves. Most nerves are mixed motor and sensory. The intrinsic psoas nerves are the only purely motor nerves and the lateral femoral cutaneous nerve is the only purely sensory nerve.

\subsubsection{Motor nerves}

The femoral nerve is a mixed motor and sensory nerve that arises from the lateral border of the psoas muscle. It has two divisions, anterior and posterior. The anterior division gives off the anterior cutaneous nerve and muscular branches. It gives motor innervation to the pectineus and sartorius muscles. The posterior division gives off the saphenous nerve (sensory) and muscular branches. It gives motor innervation to the quadriceps femoris, which is composed of the rectus femoris, vastus lateralis, vastus medialis, and vastus intermedius.

The obturator nerve is a mixed motor and sensory nerve that arises from the medial border of the psoas muscle. It innervates the adductor muscles of the lower extremity. These include the external obturator, adductor longus, adductor brevis, adductor magnus, gracilis, and the pectineus (inconstant) muscles. It does not innervate the obturator internus. It also supplies the sensory innervation of the skin of the medial aspect of the proximal thigh.

\subsubsection{Sensory nerves}

The ilioinguinal nerve innervates the skin at the base of the penis and upper scrotum in males and the skin of the mons pubis and labia majora in females. 
The iliohypogastric nerve consists of two branches that innervate the skin of the lower abdominal wall. The lateral cutaneous branch innervates the skin of the gluteal region. Of note, this nerve can also be injured when harvesting an anterior iliac crest bone graft. The anterior cutaneous branch innervates the hypogastric, or lower abdominal region.

The genitofemoral nerve consists of two branches, the genital and femoral branches. The genital branch innervates the cremaster muscle and scrotal skin in males and the skin of the mons pubis and labia majora in females. The femoral branch innervates the skin over the femoral triangle. This nerve is distinct from the other sensory nerves in that it does not follow a lateral trajectory to the site of innervation, but rather emerges on the anterior surface of the psoas and descends on the ventral surface.

The lateral femoral cutaneous nerve innervates the lateral aspect of the thigh. It consists of an anterior and a posterior branch. The anterior branch innervates the skin of the anterior and lateral surfaces of the thigh, as far as the knee. The posterior branch innervates the lateral and posterior surfaces of the thigh, from the level of the greater trochanter to the middle of the thigh.

The anterior femoral cutaneous nerve innervates the anterior and medial aspect of the thigh.

\subsection{Safe zones}

Early anatomic work related to the retroperitoneal transpsoas approach by Moro et al. helped to establish a safety zone to prevent nerve injuries when operating (Moro, et al., 2003). Specifically, they found that it was safe to traverse the psoas muscle at levels L4/5 and above, with the exception of the genitofemoral nerve, which is at risk at between L3 and L4.

Further studies described the course of the plexus, and found that the plexus lies within the substance of the psoas muscle between the junction of the transverse process and vertebral body, while exiting along the medial edge of the psoas distally (Benglis, et al., 2009). It is most dorsally positioned at the posterior endplate of L1/2 with a general trend of progressive ventral migration down to the level of L4/5. When a ratio of the distance from the posterior vertebral body wall to the total disc space length was calculated, it was found that there was a $0,0.11,0.18$, and 0.28 ratio for $\mathrm{L} 1 / 2, \mathrm{~L} 2 / 3, \mathrm{~L} 3 / 4$, and $\mathrm{L} 4 / 5$, respectively. These findings suggest that an overly posterior placement of the dilator and/or retractor can lead to nerve injuries, especially at L4/5, where the ventral migration is nearly one third of the disc space from the posterior vertebral body wall.

A cadaveric study by Uribe et al. established four different zones and described safe working zones for MIS LIF (Uribe, et al., 2010) (Figure 1). The four zones represent different quartiles of the vertebral body, with zone I representing the most anterior and zone IV representing the most posterior quartile. The lumbar plexus, along with nerve roots, lie within the substance of the psoas muscle and dorsal to zone IV. The genitofemoral nerve was the only structure found to be ventral to zone III, starting at L2/3 and progressing caudally to L3/4 and L4/5.

It was determined that the safe anatomical zones to avoid nerve injury from L1/2 to L3/4 are the midpoint of zone III (posterior third of the disc space), and the safe zone for L4/5 is at the zone II/III junction (mid disc space). The genitofemoral nerve is at risk in zone II at 


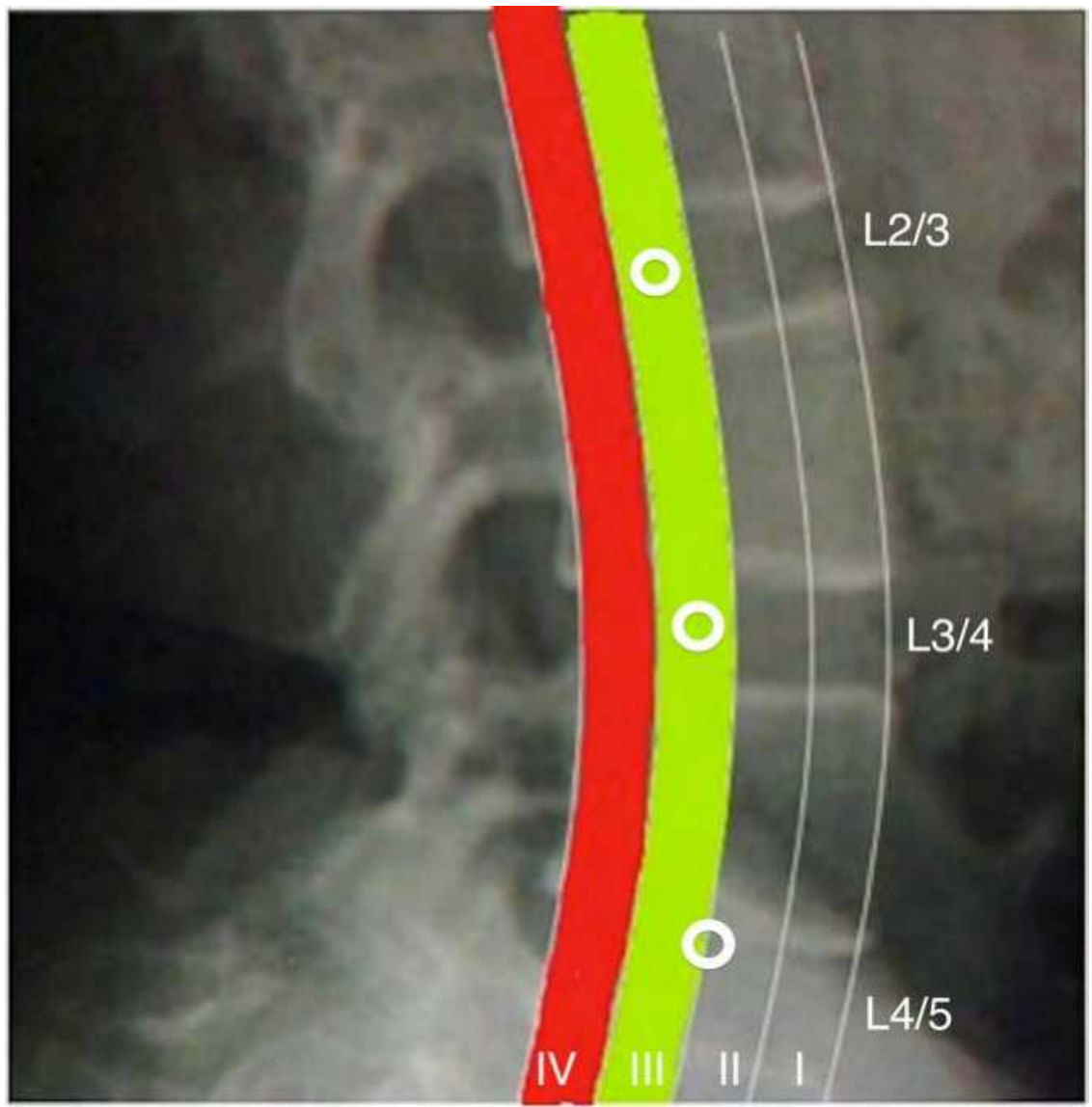

Fig. 1. Safe Anatomical Zones for MIS LIF. There are four quartiles, I-IV, from anterior to posterior. The open circles indicate a "safe zone" for placement of the retractor and for subsequent exposure. From L1/2 to L3/4, the posterior third is generally safe. At L4/5, placement at the midpoint between zone II and III is generally safe since this will decrease the risk of injuring the femoral nerve.

L2/3 and in zone I at L3/4 and L4/5. The ilioinguinal, iliohypogastric, and lateral femoral cutaneous nerves in the retroperitoneal space are also at risk since they travel obliquely, inferiorly, and anteriorly to the reach the iliac crest and the abdominal wall outside of the psoas in the retroperitoneal space.

There is a chance of lumbar plexus injury even in the early stages of the operation while obtaining access to the retroperitoneal space. Four nerves, the subcostal, iliohypogastric, ilioinguinal, and lateral femoral cutaneous nerves, are at risk of injury at this stage of the operation.

In addition to nerve injury, visceral and vascular structures should also be considered. The importance of meticulous preoperative planning was illustrated by Regev et al. in their 
morphometric study looking at the relationship of vascular structures as it relates to MIS LIF, where they found that the safe corridor for performing a discectomy and intervertebral cage placement progressively narrows from L1/2 to L4/5 (Regev, et al., 2009). In the presence of scoliosis, these corridors can potentially be further narrowed. One should also keep in mind that the kidneys are in the retroperitoneal space.

\section{Surgical technique}

The technique of the retroperitoneal transpsoas MIS LIF by our team has evolved with time and experience. Significant changes were made to our technique in 2010, and these changes have been the standard method we currently use for every patient. Specifically, the technique below refers to use of the XLIF® procedure. In general, the main principles apply to any lateral access system; however, a significant difference that will not apply to other systems is the use of a directional, triggered-EMG, which will be explained further in this discussion.

\subsection{Preoperative planning and positioning}

The preoperative planning is critical to ensure that the patient is a good surgical candidate. Preoperative magnetic resonance imaging (MRI) is evaluated to ensure that abdominal blood vessels will not hinder access to the desired disc space. A preoperative AP $\mathrm{x}$-ray is evaluated to determine which side will provide the best access to the desired level, especially at L4/5, in relation to the iliac crest (Figure 2).

The patient is then placed in the lateral position with the optimal side facing up. If a scoliotic deformity is present, the patient is placed with the concave side facing up. The reasoning for this is that this usually provides better access to the L4/5 disc space if that it is an operative level. In addition, positioning the concave side up will allow for access to multiple levels through potentially fewer and smaller incisions.

At our institution, patients are placed on a $\mathrm{Cmax}{ }^{\circledR}$ table (Steris, Mentor, $\mathrm{OH}$ ), but any radiolucent operating table that allows for adjustment of flexion, extension, Trendelenburg/reverse Trendelenburg, as well as lateral tilting will suffice. The iliac crest is placed at the level of the table break where table flexion occurs. The legs are flexed maximally at the knee and hip to relax tension on the psoas muscle. A roll is placed beneath the axilla to prevent brachial plexus injury, and a roll is placed under the iliac crest to promote flexion at the iliac crest for improved access to the L4/5 level.

Intraoperative fluoroscopy is then used to position the patient in such a manner that a symmetric AP image with the pedicles equidistant from the spinous processes is achieved. It is essential that these images be as accurate and symmetric as possible to prevent inadvertently dissecting too far anteriorly or posteriorly. Caution should be exercised if a prior laminectomy exists over the desired level and spinous processes cannot be visualized.

Once properly positioned, the patient is taped and secured into place at the iliac crest and chest. The ipsilateral hip and leg are then taped to pull the iliac crest inferiorly and then secured to the table to prevent the patient from moving during surgery. The patient is then taped and secured into position (Figure 3). 


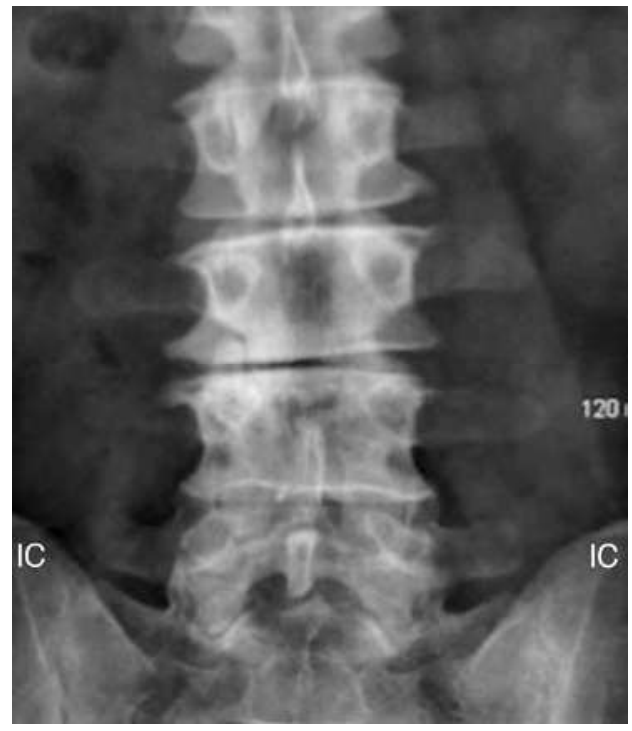

Fig. 2. AP radiograph of the Lumbar Spine. It is crucial to evaluate the clearance of the iliac crest (IC) preoperatively to determine positioning and operative feasibility.

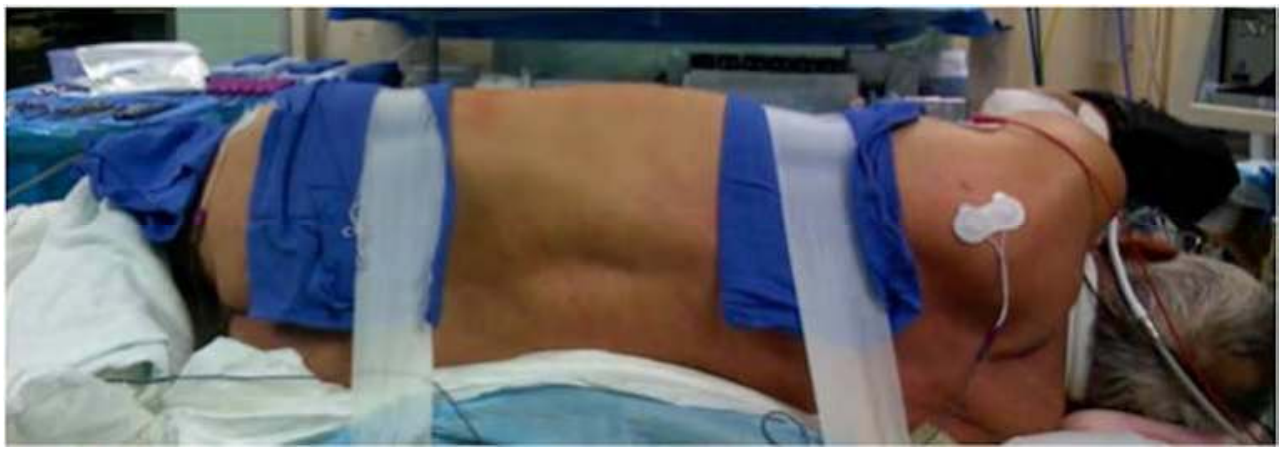

Fig. 3. Lateral Decubitus Positioning for MIS LIF. The patient is placed on an adjustable operative table and secured with silk tape. There is a bend in the table to allow for better access during surgery.

A repeat $\mathrm{AP}$ fluoroscopic image is again taken to ensure that good images are still obtainable and the bed is tilted slightly if correction of the image is needed. The relationship of the ipsilateral iliac crest and the lowest level to be approached are then evaluated. The angle of the disc space in relation to the iliac crest should ideally allow direct access to the disc space. At this point, the table is flexed at the level of the iliac crest just enough to give access to the disc space. If there is good access to the disc space without needing to flex the table, then it is advisable to perform the operation without flexing the table. Too much flexion of the table can put tension on the lumbar plexus and potentially cause nerve injury, so the table is flexed as minimally as possible while still achieving good access to the disc space. 
Lateral fluoroscopy is then used, and the patient's position is modified with Trendelenburg or reverse Trendelenburg to obtain images clearly displaying endplates, posterior vertebral cortex, pedicle, as well as to evaluate the relationship of the disc space of interest to the ipsilateral iliac crest. A guide wire can be placed on the patient's skin to localize the plane of the disc space. Palpation of this area confirms that the iliac crest will not obstruct the pathway to the disc space.

An AP image for final positioning is then obtained to ensure there has not been any significant patient movement and that the images are still acceptable. Lateral fluoroscopy is then obtained to mark the disc space transversely and the posterior third of the disc space vertically. An exception is at L4/5, where the vertical mark is at the middle of the disc space based on the anatomic safe zones (Uribe, et al., 2010). If one level is to be approached, a single transverse incision approximately $5 \mathrm{~cm}$ is used. If more than one level is to be approached, a single vertical incision or multiple transverse incisions are used depending on the length of the incisions and cosmetic concerns.

\subsection{Operative procedure}

The area is then prepped and draped. An incision is made with a \#10 scalpel blade to the subcutaneous fat. A second posterior incision is routinely not used as originally described (Ozgur, et al., 2006), as this route of access may cause injury to the ilioinguinal or iliohypogastric nerves (Uribe, et al., 2010). A self-retaining retractor is used to help dissect subcutaneous fat transversely along the original incision line with monopolar cautery until fascia is encountered. A transverse incision is then made in the fascia with monopolar cautery in line with the disc space. If multiple disc spaces are being approached, separate fascial incisions are made for each disc space to help stabilize the retractor.

Once the fascial incision over the area of interest is completed and muscle is encountered, two tonsil hemostats are used to dissect through muscle gently in the plane of the disc space through as small of an access as possible. Great care is taken to ensure the dissection is performed in line with the original skin marking for the posterior third of the disc space (or at the mid-vertebral body at L4/5) and that the dissection is not carried too anteriorly (to avoid bowel injury) or too posteriorly (to avoid nerve injury). The external oblique, internal oblique, and transversus abdominus muscles are identified and dissected until the transversalis fascia and retroperitoneal space are encountered.

Once in the retroperitoneal space, the quadratus lumborum can be palpated posterolaterally. The quadratus can then be followed medially until the transverse process of the vertebra of interest can be palpated. Then proceeding further medially, the psoas muscle can be palpated.

The first dilator can be inserted at this point, guided with the surgeon's finger anterior to the dilator to avoid peritoneal injury. The dilator is docked gently on the psoas without traversing the psoas. Lateral fluoroscopy is obtained to check position of the dilator to ensure it is in correct position (posterior third of the disc space, except for L4/5, in which case the middle of the disc space is the target) and the dilator position is adjusted as needed. The dilator is stimulated for triggered EMG (t-EMG) and the dilator is then rotated $360^{\circ}$ to check for activity. Typically, anything $\geq 11 \mathrm{~mA}$ indicates a safe distance from any surrounding neural structure (Table 1). The dilator is then advanced through the 
psoas muscle until it is docked onto the spine. The dilator is again stimulated for t-EMG and rotated $360^{\circ}$ to check for activity. Lateral fluoroscopy is again obtained to confirm position of the dilator in relation to the disc space as mentioned previously. If the stimulation of the initial dilator did not reveal any concerning t-EMG responses, a guide wire is placed through the dilator into the disc space to maintain position. Sequential dilators are then used to dilate the psoas muscle and stimulated in a similar fashion as described above. Once the final dilator is placed, it is stimulated for t-EMG and the responses are analyzed.

\begin{tabular}{|c|cc|}
\hline $\begin{array}{c}\text { Numeric } \\
\text { Reading }(\mathbf{m A})\end{array}$ & Color Displayed & Interpretation \\
\hline $\mathbf{2 1 1}$ & Green & Acceptable \\
\hline $\mathbf{5 - 1 0}$ & Yellow & Caution \\
\hline$<5$ & Red & Alert \\
\hline
\end{tabular}

Table 1. Triggered EMG interpretation.

Sharp decreases in the threshold are not uncommon at this portion of the procedure. In fact, finding these sharp decreases are advantageous. The position of the femoral nerve can be estimated by the location of the sharp decreases in the t-EMG threshold. Ideally, the sharp decreases will be present when stimulating with the dilator posteriorly and increased thresholds present anteriorly; thus the femoral nerve can be estimated to be posterior to the dilators. This orientation will allow placement and opening of the retractor with minimal risk of nerve injury. If decreased thresholds are obtained anteriorly, the guide wire and dilators are removed and advanced more anteriorly so that the dilators are positioned anterior to the femoral nerve. The sequence for dilator and guide wire placement described above is again carried out.

Once the t-EMG stimulation with the final dilator verifies decreased threshold responses posteriorly and increased threshold responses anteriorly, the retractor is then placed over the dilators with the retractor blades oriented superiorly, inferiorly, and posteriorly. Downward pressure is applied to the retractor during the procedure until final placement of the shim blade to prevent psoas muscle fibers from creeping into the surgical field. The retractor is locked into place with the articulating arm while maintaining downward pressure. The dilators are removed while the guide wire is kept in place. A light source is attached to the inferior blade and used in conjunction with suction to visualize the disc space while maintaining downward pressure on the retractor. The surgeon should be able to visualize "red \& white" indicating the disc space and small amounts of psoas muscle fibers that have crept into the surgical field. If only "red" is seen, then too much psoas muscle is in the field to visualize the disc space. If only "white" is seen, it is possible that the fascia of the psoas muscle has not been penetrated, which may cause the retractor to shift if it slides off the fascia during the procedure. The field is also inspected for evidence of nerves that could be injured with the procedure. Anything suspicious for being a nerve is stimulated with manual t-EMG to check for EMG activity. Sensory nerves will not stimulate with t-EMG, so a high index of suspicion must be maintained for an object that does not trigger EMG response. 
Once it is confirmed that disc space is visualized and no nerves are present in the surgical field, lateral x-ray is obtained to check the position of the retractor in relation to the disc space. The shim blade is engaged into the posterior blade of the retractor but not deep into the disc space yet.

The retractor, while maintaining downward pressure, is adjusted into the correct position. The articulating arm is loosened and moving the retractor in relation to the guide wire helps to maintain proper orientation. Once proper position is attained on lateral fluoroscopy, AP fluoroscopy is used to show the superior-inferior relation of the retractor blades to the disc space. The shim blade is advanced into the disc space and malleted into position firmly with image guidance using AP fluoroscopy. The articulating arm is then attached and tightened to lock the retractor into position. Lateral fluoroscopy is then used to "look down" the posterior blade to ensure the proper pathway for the remainder of the procedure. The manual t-EMG stimulator is used to stimulate the entire surgical field and behind the posterior blade. Decreased thresholds elicited posterior to the posterior blade are expected and desired to ensure the working area is anterior to the femoral nerve, which is now protected by the posterior blade. The guide wire is then removed.

Once the retractor is in final position, the rest of the procedure must be performed as efficiently and quickly as possible to reduce the duration of retraction of the lumbar plexus. The retractor is now opened minimally to just allow discectomy and placement of the interbody graft.

The position of the anterior longitudinal ligament can be estimated by visualizing the slope of the anterior vertebral body. The procedure should remain as posterior as possible to this slope to prevent unwanted rupture of the anterior longitudinal ligament. A wide rectangular annulotomy is then made with an annulotomy knife. A pituitary rongeur is then used to remove disc material. A curved Cobb elevator is placed into the disc space with the handle vertically oriented and malleted under AP fluoroscopy guidance until the contralateral annulus is broken. This procedure is repeated with the curve of the Cobb elevator in the opposite orientation. The box cutter disc shaver is then placed in the disc space. Vertical orientation of the handle is confirmed and the box cutter is malleted flush with the posterior blade under AP fluoroscopy guidance to ensure the endplates are not violated. Once the box cutter is removed, AP fluoroscopy is used to confirm position of the shim blade in the disc space, which can be malleted into the disc space to guarantee the stability of the retractor. Again, a pituitary rongeur is used to remove disc material.

Depending on the preoperative x-ray, a straight or lordotic poly-ether-ether-ketone (PEEK) interbody cage can be filled with a variety of biologics. Our practice now is to pack approximately $5 \mathrm{cc}$ of cadaveric cancellous bone mixed with mesenchymal stem cells (Osteocel Plus ${ }^{\circledR}, \mathrm{NuVasive}$, San Diego, CA) into the cage. A graft retainment device is used to retain the packed contents in the cage, and the cage is then placed in the disc space with a vertical orientation of the handle. It is malleted into position until the medial radiographic marker in line with the spinous process. The graft is then released and the retainment device removed. The surgical field is inspected for any graft that may have become dislodged during placement, and removed if idenified. The area is inspected for any bleeding and bipolar cautery can be used to obtain hemostasis. The articulating arm is loosened and the 
retractor is then closed. The retractor is removed slowly from the surgical field while inspecting for any bleeding.

Once the retractor is completely removed, final $\mathrm{AP}$ and lateral fluoroscopic images are obtained to ensure proper placement of the graft. The operating table is then leveled to assist with incision closure. Fascia is closed with interrupted 0 Vicryl sutures and the subcutaneous layer closed with 3-0 Vicryl sutures. The skin is approximated with 4-0 subcuticular Monocryl suture and dressed with Dermabond $\AA$.

\section{Biomechanics}

\subsection{PEEK interbody cage}

An essential component of MIS LIF is the placement of a large interbody cage. Traditionally, implant materials have been autograft or allograft bone, but issues with fracture, migration, and pseudoarthrosis led to the development of synthetic cages such as titanium, carbon fiber, and PEEK (Yang, et al., 2011). Among the synthetic cage materials, PEEK has been found to be favorable since it shares the same modulus of elasticity as bone (Brantigan \& Steffee, 1993; Matge, 2002; Cho, et al., 2004). In addition, it is also non-absorbable, elicits a minimal cellular response, and allows for a clear, unobstructed view of new bone formation during follow-up exams (Boakye, et al., 2005; Vaidya, et al., 2008).

The placement of a large interbody cage, as accommodated by the lateral approach, is an advantage of MIS LIF. Large-diameter solid implants are less likely to subside compared to small-diameter cages, possibly related to a more efficient transfer of force to the endplate (Pearcy, et al., 1983; Closkey, et al., 1993; Lowe, et al., 2004).

\subsection{Lateral plate}

The MIS LIF can be supplemented with a lateral plate that spans across the disc space (Figure 4). The titanium plate has a rostral and caudal screw hole, and it can come in varying lengths (there is also a four-screw hole type, which we do not routinely use due to its larger profile). It is seated on two bicortical titanium screws that are placed across the width of the vertebral body parallel to the adjacent endplate.

Biomechanical comparisons between the lateral plate and stand-alone, unilateral pedicle screw, and bilateral pedicle screw constructs have demonstrated its increased rigidity compared to a stand-alone construct to promote arthrodesis (Bess, et al., 2008; Cappuccino, et al., 2010). The greatest biomechanical advantage of a lateral plate is its very favorable range of motion restriction in lateral bending, with only bilateral pedicle screws offering slightly more rigidity. In total, however, lateral plates still fall short of unilateral and bilateral pedicle screws, which are much more rigid overall.

Good candidates for a lateral plate supplementation should be free of any significant gross instability, since bilateral pedicle screws would be best in that situation. For similar reasons, lateral plates may not be optimal for deformity correction. In addition, bilateral pedicle screws are preferred in this situation because lateral plates only stabilize one segment at a time compared to multilevel stabilization offered by a unified, multilevel, pedicle screw and rod construct. 


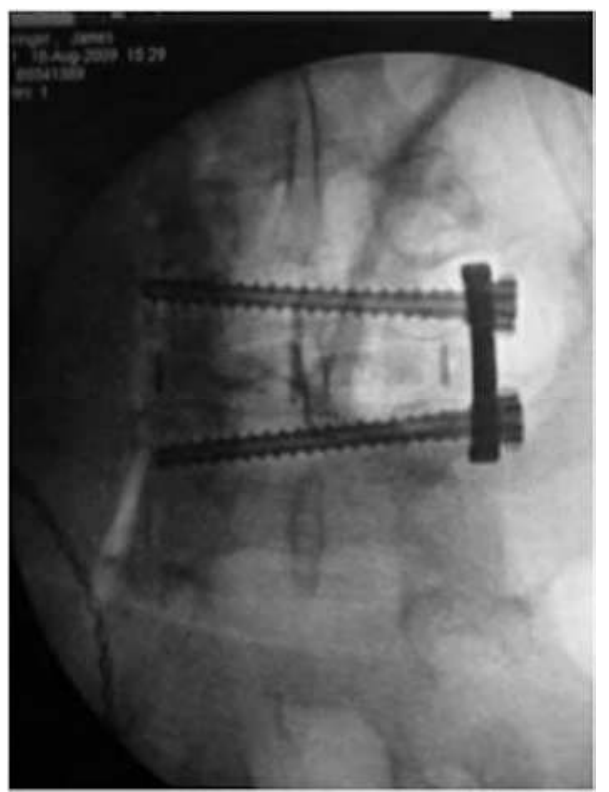

Fig. 4. Lateral Plate Fixation. This is an AP fluorographic view. Note the intervertebral cage placed spanning the entire vertebral body. The screws are placed near the subchondral bone.

\section{Applications of the lateral approach}

Surgical indications could include trauma, adult degenerative scoliosis, degenerative disc disease, spondylosis with instability, lumbar stenosis, spondylolisthesis, and adjacent segment failure. Early outcome studies have demonstrated that MIS LIF is associated with shorter OR times, minimal blood loss, few complications, minimal hospital length of stay, and quicker recovery (Dakwar, et al., 2010; Youssef, et al., 2010). Long-term outcomes are generally favorable, with maintained improvements in patient-reported pain and function scores as well as radiographic parameters, including high rates of fusion.

\subsection{Degenerative spine disease and deformity}

Minimally invasive techniques are increasingly used to treat degenerative spine disease and deformity. The factors that make MIS LIF appealing as mentioned above are an obvious draw to surgeons trying to minimize the morbidity associated with traditional open deformity correction (Carreon, et al., 2003; Okuda, et al., 2006). Using this technique, coronal Cobb angles can be improved (Anand, et al., 2010; Dakwar, et al., 2010; Tormenti, et al., 2010; Wang \& Mummaneni, 2010) The effects on sagittal Cobb angles, such as with lumbar lordosis and the overall global sagittal balance, have not been as well established, however. This is an important topic since a positive global sagittal imbalance is most closely linked to a decreased quality of life, health status outcomes, and function (Schwab, et al., 2010). Sagittal imbalance can lead to higher energy requirements to stand and ambulate, leading to early fatigue, intolerance to standing, and walking with compensation through other joints. 
The clinical outcomes data regarding deformity correction are encouraging thus far, with improved radiographic parameters as well as improved clinical results with a lower complication profile compared to traditional open approaches (Mundis, et al., 2010).

As the role of MIS LIF in spinal deformity correction is further clarified through further research, it is important to keep in mind that the ultimate end goal should still be to reestablish spinopelvic harmony, or the proportional relationships of one regional parameter to another as it relates to global spinopelvic alignment, as spinopelvic harmony has been directly linked to a satisfactory postsurgical outcome as assessed by health related quality of life instruments (Schwab, et al., 2010; Lafage, et al., 2011). Three basic radiographic targets to aim for in order to achieve spinopelvic harmony include: 1) sagittal vertical axis of $<50 \mathrm{~mm}$ or T1-SI $<0^{\circ}, 2$ ) pelvic tilt of $<20^{\circ}$, and 3) lumbar lordosis = pelvic incidence $\pm 9^{\circ}$ (Schwab, et al., 2010). Attention to these three goals serve as the foundation for individual, patientspecific spinopelvic realignment in the sagittal plane, and even partial improvements of these parameters may translate to better clinical outcomes.

Adjacent segment failure is a common complication encountered in practice in patients with prior lumbar fusions. Operations to address this issue can often involve further posterior muscle dissection and revision of the existing instrumentation, all while negotiating through previous scar tissue, leading to risks of infection and CSF leaks. The MIS LIF is an option for treatment of adjacent segment failure. A virgin corridor is traversed, with placement of an intervertebral cage, which avoids some of the pitfalls of reoperations as mentioned above. In addition, if further internal fixation is desired, then a lateral plate could be placed without much additional difficulty. Literature regarding the specific use of the lateral retroperitoneal transpsoas approach is lacking for adjacent segment failure revision surgeries, but studies related to revision surgery using a this approach for revision and explantation of lumbar total disc replacements have shown its effectiveness and low rate of complications by avoiding a previous, scarred approach (Wagner, et al., 2006; Leary, et al., 2007; Patel, et al., 2008).

\subsection{Trauma}

Another area where there has been increased interest for the use of the lateral approach is trauma. Traumatic burst fractures commonly occur in the lumbar spine, with many occurring at the thoracolumbar junction. The decision of whether or not to treat with nonoperative management with external orthoses or bedrest versus surgical decompression, instrumentation, and fusion is beyond the scope of this discussion. However, when surgical treatment is planned for situations where there is instability with neurologic deficit, a minimally invasive retroperitoneal transpsoas approach is an option.

In a report by Smith et al. with a follow-up of two years, patients treated with lateral corpectomies with supplemental instrumentation were found to have very favorable OR times, estimated blood loss, and hospital length of stay (Smith, et al., 2010). None of the patients required reoperations, and there was a significant improvement in the neurologic status based on the American Spinal Injury Association categorization, with none experiencing a neurologic decline. 


\section{Complications}

As with any operation, there will always be a risk of complications that underscore the importance of meticulous attention to detail throughout the perioperative period (Knight, et al., 2009). Complications can arise from the result of inadequate preoperative planning. For instance, neurovascular structures may be in the way of the intended exposure, which may preclude a safe corridor for operating. Close attention to preoperative MRI's can help avoid this from happening. In addition, positioning mistakes leading to placement of the nonoptimal side positioned up can make access to the L4/5 disc space, for example, much more difficult, leading to an increased risk of postoperative motor or sensory deficits.

\subsection{Numbness, paresthesia, and weakness}

The lateral retroperitoneal transpsoas approach is a technique that can be challenging since it is a non-traditional approach for many spine surgeons who are more accustomed to a posterior approach. Because of this, it does have a learning curve, and the skill at which it is performed is very dependent on experience with the regional anatomy and with the approach itself. Small changes in technique with this approach can result in dramatic changes in patient outcome due to the proximity of the lumbar plexus. Real-time EMG monitoring is critical to minimize the chance of motor nerve injury (Uribe, et al., 2010). However, sensory nerves cannot be monitored, thus leaving them susceptible to iatrogenic injury if there is not a thorough understanding of the regional anatomy.

Nerve injuries can lead to motor and sensory deficits, with the highest rates with L4/5 interbody approaches. The current literature is inconsistent with its reporting of postoperative "thigh" symptoms, which could range from numbness, paresthesias, dysesthesias, or weakness. Because of this, an overall rate of "thigh complications" ranging from $0.7 \%-62.7 \%$ must be considered with a fair degree of caution (Knight, et al., 2009; Cummock, et al., 2011; Rodgers, et al., 2011).

When looking specifically at the type of complication, the rate of paresthesias following MIS LIF can range from 0.7\% to 30\% (Bergey, et al., 2004; Knight, et al., 2009; Cummock, et al., 2011; Rodgers, et al., 2011), and numbness has been reported in 8.3\% - 42.4\% (Dakwar, et al., 2010; Cummock, et al., 2011; Pimenta, et al., 2011). The specific nerve distribution may vary as well, but commonly affected nerves are the genitofemoral, lateral femoral cutaneous, and anterior femoral cutaneous nerves. It is important to distinguish between the different dermatomes of these sensory nerves on the postoperative examination, and not to simply report that a patient has thigh pain or numbness. Reports of motor weakness from femoral nerve injury have also varied, ranging from 3.4\% - 23.7\% (Knight, et al., 2009; Cummock, et al., 2011; Pimenta, et al., 2011).

It is important to realize that most motor and sensory deficits are transient and do recover, with 50\% recovery at 90 days, and 90\% recovery at 1 year (Cummock, et al., 2011). This may be a result of the muscles and nerves recovering from manipulation, inflammation, and irritation during the operation. As a result, it is advisable to fully disclose to patients preoperatively that there is a chance of motor or sensory deficit following the operation, but that the vast majority of cases are transient in nature. 


\subsection{Abdominal wall paresis}

Abdominal wall paresis, also referred to as a "pseudohernia", has been identified as a potential complication of the MIS lateral approach (Dakwar, et al., 2011). The mechanism is attributed to iatrogenic nerve injury during the initial dissection of the abdominal wall. Consequences include denervation, paresis, and bulging of the anterior abdominal wall. Associated signs and symptoms include swelling, pain, hyperesthesia, or other sensory abnormalities. If suspected, it is important to rule out a true abdominal hernia in these instances. In many cases, spontaneous recovery can occur.

\subsection{Hardware-related complications}

There have been few reports of complications attributed to the hardware implanted such as the interbody cage or lateral plate. Recently, Dua et al. reported a $15 \%$ rate of hardwarerelated complications based off a series of 13 patients (Dua, et al., 2010). These cases consisted of two atraumatic coronal plane fractures at L4/5 in the first six weeks of the postoperative period.

A review of our own series has demonstrated a hardware-related complications rate of $5.9 \%$ in a series of 101 consecutive cases (Le, et al., 2011). The complications included three hardware failures and three vertebral body fractures. All cases were atraumatic. All cases presented with recurrent back pain except one, which was identified incidentally. All hardware failures involved a dislodged lateral plate and lock nut(s).

The mechanism is unclear, but may involve cage subsidence with a fixed angle screw, resulting in the screws cutting through the vertebral bodies in a coronal plane, a stress riser in the area of stress concentration, violation of the endplate during preparation or screw insertion, or malplacement of the hardware lock nuts (Disch, et al., 2008; Dua, et al., 2010; Le, et al., 2011).

\subsection{Subsidence}

As with any technique used for lumbar fusion, subsidence of the cage can occur at one or both endplates. The subsequent progressive deformity and compression of neural elements can lead to a loss of indirect decompression and reduced chance of successful fusion and possible reoperation (Closkey, et al., 1993; Kozak, et al., 1994).

In a study that included 140 patients and 238 levels fused in the lumbar spine with a mean follow-up of 9.6 months, we have recently found subsidence to be present in $14.3 \%$ of the cases, and in $8.8 \%$ of the total levels fused (Le, et al., 2011). Only $2.1 \%$ of the patients had symptomatic subsidence, however. Subsidence appears to correlate with construct length.

The most important finding, however, was that there was a $14.1 \%$ rate of subsidence with smaller $18 \mathrm{~mm}$ cages versus only $1.9 \%$ with larger $22 \mathrm{~mm}$ cages, leading to the conclusion that the largest interbody cage should be used whenever feasible.

\subsection{Rhabdomyolysis}

Rhabdomyolysis is a rare, but known, complication of spinal surgery. In severe cases, acute renal failure may result. The first cases of rhabdomyolysis and acute renal failure have 
recently been reported following MIS LIF (Dakwar, et al., 2011). This potential complication should be suspected in appropriate cases especially in morbidly obese patients and in procedures associated with prolonged operative times.

\section{Conclusions and key points}

The retroperitoneal transpsoas approach is a safe and effective alternative to traditional posterior, open lumbar techniques. It can be utilized for a variety of clinical applications including trauma, adult degenerative scoliosis, degenerative disc disease, spondylosis with instability, lumbar stenosis, spondylolisthesis, and adjacent segment failure. As with most minimally invasive techniques, there is a learning curve to be overcome in order to minimize the risk of iatrogenic nerve injuries. An integral aspect of this curve is to always be aware of the regional anatomy encountered. It is important to stay within the "safe zones" when performing an MIS LIF, staying in the posterior third of the disc space at L1/2, L2/3, and L3/4, or at the midpoint of the vertebral body at L4/5. Directional, t-EMG can help guide the surgeon and alert of any critical distances from surrounding motor nerves. Even with this, transient sensory deficits and, on occasion, weakness may occur, and it is important to discuss this potential with surgical candidates preoperatively.

\section{References}

Anand, N., Rosemann, R., Khalsa, B., \& Baron, E. M. (2010). "Mid-term to long-term clinical and functional outcomes of minimally invasive correction and fusion for adults with scoliosis." Neurosurg Focus 28(3): E6.

Benglis, D. M., Elhammady, M. S., Levi, A. D., \& Vanni, S. (2008). "Minimally invasive anterolateral approaches for the treatment of back pain and adult degenerative deformity." Neurosurgery 63(3 Suppl): 191-196.

Benglis, D. M., Vanni, S., \& Levi, A. D. (2009). "An anatomical study of the lumbosacral plexus as related to the minimally invasive transpsoas approach to the lumbar spine." J Neurosurg Spine 10(2): 139-144.

Bergey, D. L., Villavicencio, A. T., Goldstein, T., \& Regan, J. J. (2004). "Endoscopic lateral transpsoas approach to the lumbar spine." Spine (Phila Pa 1976) 29(15): 1681-1688.

Bess, R. S., Cornwall, G. B., Vance, R., Bachus, K. N., \& Brodke, D. S. (2008). Biomechanics of lateral arthrodesis. eXtreme Lateral Interbody Fusion (XLIF). J. A. Goodrich and I. J. Volcan. St. Louis, Missouri, Quality Medical Publishing, Inc.: 31-40.

Boakye, M., Mummaneni, P. V., Garrett, M., Rodts, G., \& Haid, R. (2005). "Anterior cervical discectomy and fusion involving a polyetheretherketone spacer and bone morphogenetic protein." J Neurosurg Spine 2(5): 521-525.

Brantigan, J. W., \& Steffee, A. D. (1993). "A carbon fiber implant to aid interbody lumbar fusion. Two-year clinical results in the first 26 patients." Spine (Phila Pa 1976) 18(14): 2106-2107.

Cappuccino, A., Cornwall, G. B., Turner, A. W., Fogel, G. R., Duong, H. T., Kim, K. D., \& Brodke, D. S. (2010). "Biomechanical analysis and review of lateral lumbar fusion constructs." Spine (Phila Pa 1976) 35(26 Suppl): S361-367.

Carreon, L. Y., Puno, R. M., Dimar, J. R., 2nd, Glassman, S. D., \& Johnson, J. R. (2003). "Perioperative complications of posterior lumbar decompression and arthrodesis in older adults." J Bone Joint Surg Am 85-A(11): 2089-2092. 
Cho, D. Y., Lee, W. Y., \& Sheu, P. C. (2004). "Treatment of multilevel cervical fusion with cages." Surg Neurol 62(5): 378-385, discussion 385-376.

Closkey, R. F., Parsons, J. R., Lee, C. K., Blacksin, M. F., \& Zimmerman, M. C. (1993). "Mechanics of interbody spinal fusion. Analysis of critical bone graft area." Spine (Phila Pa 1976) 18(8): 1011-1015.

Cloward, R. B. (1953). "The treatment of ruptured lumbar intervertebral discs by vertebral body fusion. I. Indications, operative technique, after care." J Neurosurg 10(2): 154168.

Cummock, M. D., Vanni, S., Levi, A. D., Yu, Y., \& Wang, M. Y. (2011). "An analysis of postoperative thigh symptoms after minimally invasive transpsoas lumbar interbody fusion." J Neurosurg Spine 15(1): 11-18.

Dakwar, E., Cardona, R. F., Smith, D. A., \& Uribe, J. S. (2010). "Early outcomes and safety of the minimally invasive, lateral retroperitoneal transpsoas approach for adult degenerative scoliosis." Neurosurg Focus 28(3): E8.

Dakwar, E., Le, T. V., Baaj, A. A., Le, A. X., Smith, W. D., Akbarnia, B. A., \& Uribe, J. S. (2011). "Abdominal wall paresis as a complication of minimally invasive lateral transpsoas interbody fusion." Neurosurg Focus 31(4): E18.

Dakwar, E., Rifkin, S. I., Volcan, I. J., Goodrich, J. A., \& Uribe, J. S. (2011). "Rhabdomyolysis and acute renal failure following minimally invasive spine surgery: report of 5 cases." J Neurosurg Spine 14(6): 785-788.

Dakwar, E., Vale, F. L., \& Uribe, J. S. (2011). "Trajectory of the main sensory and motor branches of the lumbar plexus outside the psoas muscle related to the lateral retroperitoneal transpsoas approach." J Neurosurg Spine 14(2): 290-295.

Disch, A. C., Knop, C., Schaser, K. D., Blauth, M., \& Schmoelz, W. (2008). "Angular stable anterior plating following thoracolumbar corpectomy reveals superior segmental stability compared to conventional polyaxial plate fixation." Spine (Phila Pa 1976) 33(13): 1429-1437.

Dua, K., Kepler, C. K., Huang, R. C., \& Marchenko, A. (2010). "Vertebral body fracture after anterolateral instrumentation and interbody fusion in two osteoporotic patients." Spine J 10(9): e11-15.

Eck, J. C., Hodges, S., \& Humphreys, S. C. (2007). "Minimally invasive lumbar spinal fusion." J Am Acad Orthop Surg 15(6): 321-329.

Harms, J., \& Rolinger, H. (1982). "[A one-stager procedure in operative treatment of spondylolistheses: dorsal traction-reposition and anterior fusion (author's transl)]." Z Orthop Ihre Grenzgeb 120(3): 343-347.

Harms, J. G., \& Jeszenszky, D. (1998). "The unilateral, transforaminal approach for posterior lumbar interbody fusion." Oper Orthop Traumatol 6: 88-99.

Hodgson, A. R., \& Stock, F. E. (1956). "Anterior spinal fusion a preliminary communication on the radical treatment of Pott's disease and Pott's paraplegia." Br J Surg 44(185): 266-275.

Knight, R. Q., Schwaegler, P., Hanscom, D., \& Roh, J. (2009). "Direct lateral lumbar interbody fusion for degenerative conditions: early complication profile." J Spinal Disord Tech 22(1): 34-37.

Kozak, J. A., Heilman, A. E., \& O'Brien, J. P. (1994). "Anterior lumbar fusion options. Technique and graft materials." Clin Orthop Relat Res(300): 45-51. 
Lafage, V., Schwab, F., Vira, S., Patel, A., Ungar, B., \& Farcy, J. P. (2011). "Spino-Pelvic Parameters After Surgery Can be Predicted: A Preliminary Formula and Validation of Standing Alignment." Spine (Phila Pa 1976) 36(13): 1037-1045.

Le, T. V., Baaj, A. A., Dakwar, E., Burkett, C. J., Murray, G., Smith, D. A., \& Uribe, J. S. (2011). "Subsidence of PEEK Intervertebral Cages in Minimally Invasive Lateral Retroperitoneal Transpsoas Lumbar Interbody Fusion." Spine (Phila Pa 1976): In Press.

Le, T. V., Smith, D. A., Greenberg, M. S., Dakwar, E., Baaj, A. A., \& Uribe, J. S. (2011). "Complications of lateral plating in the minimally invasive lateal transpsoas approach." J Neurosurg Spine: In Press.

Leary, S. P., Regan, J. J., Lanman, T. H., \& Wagner, W. H. (2007). "Revision and explantation strategies involving the CHARITE lumbar artificial disc replacement." Spine (Phila Pa 1976) 32(9): 1001-1011.

Lowe, T. G., Hashim, S., Wilson, L. A., O'Brien, M. F., Smith, D. A., Diekmann, M. J., \& Trommeter, J. (2004). "A biomechanical study of regional endplate strength and cage morphology as it relates to structural interbody support." Spine (Phila Pa 1976) 29(21): 2389-2394.

Matge, G. (2002). "Cervical cage fusion with 5 different implants: 250 cases." Acta Neurochir (Wien) 144(6): 539-549; discussion 550.

McAfee, P. C., Phillips, F. M., Andersson, G., Buvenenadran, A., Kim, C. W., Lauryssen, C., Isaacs, R. E., Youssef, J. A., Brodke, D. S., Cappuccino, A., Akbarnia, B. A., Mundis, G. M., Smith, W. D., Uribe, J. S., Garfin, S., Allen, R. T., Rodgers, W. B., Pimenta, L., \& Taylor, W. (2010). "Minimally invasive spine surgery." Spine (Phila Pa 1976) 35(26 Suppl): S271-273.

Moro, T., Kikuchi, S., Konno, S., \& Yaginuma, H. (2003). "An anatomic study of the lumbar plexus with respect to retroperitoneal endoscopic surgery." Spine (Phila Pa 1976) 28(5): 423-428; discussion 427-428.

Mundis, G. M., Akbarnia, B. A., \& Phillips, F. M. (2010). "Adult deformity correction through minimally invasive lateral approach techniques." Spine (Phila Pa 1976) 35(26 Suppl): S312-321.

Okuda, S., Miyauchi, A., Oda, T., Haku, T., Yamamoto, T., \& Iwasaki, M. (2006). "Surgical complications of posterior lumbar interbody fusion with total facetectomy in 251 patients." J Neurosurg Spine 4(4): 304-309.

Ozgur, B. M., Aryan, H. E., Pimenta, L., \& Taylor, W. R. (2006). "Extreme Lateral Interbody Fusion (XLIF): a novel surgical technique for anterior lumbar interbody fusion." Spine J 6(4): 435-443.

Patel, A. A., Brodke, D. S., Pimenta, L., Bono, C. M., Hilibrand, A. S., Harrop, J. S., Riew, K. D., Youssef, J. A., \& Vaccaro, A. R. (2008). "Revision strategies in lumbar total disc arthroplasty." Spine (Phila Pa 1976) 33(11): 1276-1283.

Pearcy, M. J., Evans, J. H., \& O'Brien, J. P. (1983). "The load bearing capacity of vertebral cancellous bone in interbody fusion of the lumbar spine." Eng Med 12(4): 183-184.

Pimenta, L. (2001). Lateral endoscopic transpsoas retroperitoneal approach for lumbar spine surgery. VIII Brazilian Spine Society Meeting, Belo Horizo te, Minas Gerais, Brazil.

Pimenta, L., Oliveira, L., Schaffa, T., Coutinho, E., \& Marchi, L. (2011). "Lumbar total disc replacement from an extreme lateral approach: clinical experience with a minimum of 2 years' follow-up." J Neurosurg Spine 14(1): 38-45. 
Regev, G. J., Chen, L., Dhawan, M., Lee, Y. P., Garfin, S. R., \& Kim, C. W. (2009). "Morphometric analysis of the ventral nerve roots and retroperitoneal vessels with respect to the minimally invasive lateral approach in normal and deformed spines." Spine (Phila Pa 1976) 34(12): 1330-1335.

Rodgers, W. B., Gerber, E. J., \& Patterson, J. (2011). "Intraoperative and early postoperative complications in extreme lateral interbody fusion: an analysis of 600 cases." Spine (Phila Pa 1976) 36(1): 26-32.

Schwab, F., Patel, A., Ungar, B., Farcy, J. P., \& Lafage, V. (2010). "Adult spinal deformity postoperative standing imbalance: how much can you tolerate? An overview of key parameters in assessing alignment and planning corrective surgery." Spine (Phila Pa 1976) 35(25): 2224-2231.

Smith, W. D., Dakwar, E., Le, T. V., Christian, G., Serrano, S., \& Uribe, J. S. (2010). "Minimally invasive surgery for traumatic spinal pathologies: a mini-open, lateral approach in the thoracic and lumbar spine." Spine (Phila Pa 1976) 35(26 Suppl): S338-346.

Tormenti, M. J., Maserati, M. B., Bonfield, C. M., Okonkwo, D. O., \& Kanter, A. S. (2010). "Complications and radiographic correction in adult scoliosis following combined transpsoas extreme lateral interbody fusion and posterior pedicle screw instrumentation." Neurosurg Focus 28(3): E7.

Uribe, J. S., Arredondo, N., Dakwar, E., \& Vale, F. L. (2010). "Defining the safe working zones using the minimally invasive lateral retroperitoneal transpsoas approach: an anatomical study." J Neurosurg Spine 13(2): 260-266.

Uribe, J. S., Vale, F. L., \& Dakwar, E. (2010). "Electromyographic monitoring and its anatomical implications in minimally invasive spine surgery." Spine (Phila Pa 1976) 35(26 Suppl): S368-374.

Vaidya, R., Sethi, A., Bartol, S., Jacobson, M., Coe, C., \& Craig, J. G. (2008). "Complications in the use of rhBMP-2 in PEEK cages for interbody spinal fusions." J Spinal Disord Tech 21(8): 557-562.

Wagner, W. H., Regan, J. J., Leary, S. P., Lanman, T. H., Johnson, J. P., Rao, R. K., \& Cossman, D. V. (2006). "Access strategies for revision or explantation of the Charite lumbar artificial disc replacement." J Vasc Surg 44(6): 1266-1272.

Wang, M. Y., Anderson, D. G., Poelstra, K. A., \& Ludwig, S. C. (2008). "Minimally invasive posterior fixation." Neurosurgery 63(3 Suppl): 197-203.

Wang, M. Y., \& Mummaneni, P. V. (2010). "Minimally invasive surgery for thoracolumbar spinal deformity: initial clinical experience with clinical and radiographic outcomes." Neurosurg Focus 28(3): E9.

Yang, J. J., Yu, C. H., Chang, B. S., Yeom, J. S., Lee, J. H., \& Lee, C. K. (2011). "Subsidence and nonunion after anterior cervical interbody fusion using a stand-alone polyetheretherketone (PEEK) cage." Clin Orthop Surg 3(1): 16-23.

Youssef, J. A., McAfee, P. C., Patty, C. A., Raley, E., DeBauche, S., Shucosky, E., \& Chotikul, L. (2010). "Minimally invasive surgery: lateral approach interbody fusion: results and review." Spine (Phila Pa 1976) 35(26 Suppl): S302-311. 


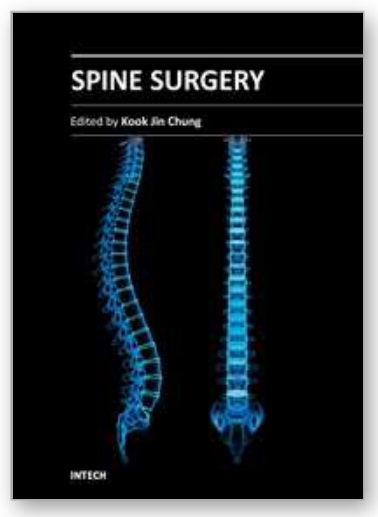

\author{
Spine Surgery \\ Edited by Dr. Kook Jin Chung
}

ISBN 978-953-51-0469-8

Hard cover, 148 pages

Publisher InTech

Published online 28, March, 2012

Published in print edition March, 2012

"Spine Surgery" is an authoritative and didactic textbook on the various fields of spine. It is written by many authors, internationally honorable experts to share their opinions with you. The chapters cover from anatomy of spine, spinal imaging technique, biology of spine, bone graft substitute, minimally invasive spinal surgery to even spinal deformity. It has many up to date results to help readers including university graduate students, medical instrumentation developers, and medical professionals including orthopaedic and neurosurgeons, rehabilitative professionals. The readers are provided with precious information and valuable guide in your daily practice.

\title{
How to reference
}

In order to correctly reference this scholarly work, feel free to copy and paste the following:

Tien V. Le and Juan S. Uribe (2012). The Minimally Invasive Retroperitoneal Transpsoas Approach, Spine Surgery, Dr. Kook Jin Chung (Ed.), ISBN: 978-953-51-0469-8, InTech, Available from:

http://www.intechopen.com/books/spine-surgery/the-minimally-invasive-lateral-retroperitoneal-transpsoasapproach

\section{INTECH}

open science | open minds

\section{InTech Europe}

University Campus STeP Ri

Slavka Krautzeka 83/A

51000 Rijeka, Croatia

Phone: +385 (51) 770447

Fax: +385 (51) 686166

www.intechopen.com

\section{InTech China}

Unit 405, Office Block, Hotel Equatorial Shanghai

No.65, Yan An Road (West), Shanghai, 200040, China 中国上海市延安西路65号上海国际贵都大饭店办公楼 405 单元

Phone: $+86-21-62489820$

Fax: $+86-21-62489821$ 
(C) 2012 The Author(s). Licensee IntechOpen. This is an open access article distributed under the terms of the Creative Commons Attribution 3.0 License, which permits unrestricted use, distribution, and reproduction in any medium, provided the original work is properly cited. 